\title{
ADIÇÃO DE APARAS DE PAPEL RECICLÁVEL NA FABRICAÇÃO DE CHAPAS DE MADEIRA AGLOMERADA
}

\author{
ADDING RECYCLABLE PAPER IN THE MANUFACTURING OF PARTICLEBOARD \\ Leandro Calegari ${ }^{1} \quad$ Clovis R. Haselein ${ }^{2} \quad$ Marcos V. Barros $^{3} \quad$ Tobias L. Scaravelli $^{4}$ \\ Lourdes Patricia E. Dacosta ${ }^{5}$ Cristiane Pedrazzi ${ }^{3}$ Everton Hillig ${ }^{6}$
}

\section{RESUMO}

Neste trabalho, foram analisadas as propriedades físico-mecânicas de chapas aglomeradas produzidas com dois tipos de papel reciclável (papel jornal e offset) e partículas de madeira de Pinus elliottii Engelm. As aparas de papel foram usadas no colchão em dois diferentes formatos (tiras ou moídas) e em diferentes proporções: $0,20,40,60$ e 100\% do peso seco das partículas. Utilizoaram-se $10 \%$ de adesivo à base de tanino-formaldeído na proporção 100:10. O experimento foi estabelecido segundo modelo fatorial, com três repetições. Os testes físico-mecânicos das chapas foram realizados segundo a norma ASTM D1037 (1995). Chapas aglomeradas produzidas tanto pela inclusão de papel jornal quanto offset no formato de tiras apresentaram tendência ao estouro durante a abertura dos pratos da prensa, causado pelo alto teor de umidade do colchão. A inclusão de aparas de papel prejudicaram a maioria das propriedades analisadas, fazendo com que não atendessem aos valores estabelecidos pelas normas DIN 68761 (1)-1961, (3)-1971 e ANSI A 208.1 (1987). A absorção d'água após 24 horas de imersão, a ligação interna e a resistência ao arrancamento de parafusos foram as propriedades mais prejudicadas. De modo geral, o uso de papel jornal se mostrou mais promissor do que o papel offset.

Palavras-chave: chapas aglomeradas; papel reciclável; tanino-formaldeído.

\begin{abstract}
In this work, there were analyzed the properties of particleboards produced with two types of recycled paper (newspaper and offset) and slash pine wood particles (Pinus elliottii Engelm.). The paper was introduced into the mattress in two forms: strands and after hammer milling, in different proportions: 0, 20, 40, 60 and $100 \%$ of ovendry weight of the particles. The adhesive used was 10\% tannin-formaldehyde (100:10 parts by weight). The experiment was set according to a factorial model, with three replications. The tests were conducted following ASTM D1037 (1995). Boards produced both with offset and newspaper strand inclusions showed tendency to blow at press opening. The inclusion of paper caused decrease in the properties, in such degree that boards did not meet commerce standards DIN 68761 (1)-1961, (3)-1971 and ANSI A 208.1 (1987). Water absorption after 24 hours, internal bond and screw whitdraw were the properties most affected. In general, the use of newspaper was more encouraging than offset paper.
\end{abstract}

Key words: particleboard; recycled paper; tannin-formaldehyde.

\section{INTRODUÇÃO}

Na comparação entre Brasil e Estados Unidos, percebe-se que, apesar de nossa situação ser menos

1. Engenheiro Florestal, Mestrando pelo Programa de Pós-Graduação em Engenharia Florestal, Centro de Ciências Rurais, Universidade Federal de Santa Maria, CEP 97105-900, Santa Maria (RS). Bolsista do CNPq. leandrocalegari@yahoo.com.br.

2. Engenheiro Florestal, PhD., Professor Adjunto do Departamento de Ciências Florestais, Centro de Ciências Rurais, Universidade Federal de Santa Maria, CEP 97105-900, Santa Maria (RS). haseleic@ ccr.ufsm.br

3. Engenheiro(a) Florestal, Mestrando(a) pelo Programa de Pós-Graduação em Engenharia Florestal, Centro de Ciências Rurais, Universidade Federal de Santa Maria, CEP 97105-900, Santa Maria (RS).

4. Acadêmico do Curso de Graduação em Engenharia Florestal, Centro de Ciências Rurais, Universidade Federal de Santa Maria, CEP 97105-900, Santa Maria (RS). Bolsista BIC-FAPERGS.

5. Engenheira Florestal, MSc., Doutoranda pelo Programa de Pós-Graduação em Engenharia Florestal, Centro de Ciências Rurais, Universidade Federal de Santa Maria, CEP 97105-900, Santa Maria (RS). Bolsista do CNPq.

6. Engenheiro Florestal, MSc., Professor da Universidade de Caxias do Sul, Rua Giacomo Batassini, 180, apartamento 302A, Bairro Santa Catarina, CEP 95030-190, Caxias do Sul (RS). ehillig@ucs.br

Recebido para publicação em 3/9/2003 e aceito em 6/5/2004. 
grave, o Brasil já apresenta os fatores que tornam necessários investimentos em reciclagem. Os principais são a falta de aterro sanitário, a reação popular frente à abertura de novos aterros e as crises freqüentes de matéria-prima virgem. Calderoni (1998) cita que a reciclagem traz um ganho anual de 1,2 bilhões de dólares para o Brasil. Esse ganho se deve, principalmente, à economia de matéria-prima, energia e água nos processos de produção. Silva e Oliveira (1999) estabelecem que, no Brasil, a composição média dos papéis no lixo entre os materiais recicláveis é de $41 \%$ em relação a outros materiais. Já para Cencig (2001), o Brasil recicla 37\% do papel que emprega, estando dentro da média mundial.

A Secretaria Municipal da Saúde e Meio Ambiente do município de Santa Maria, RS possui cadastradas seis associações de catadores de resíduos sólidos, as quais selecionam mensalmente 18 toneladas de papel reciclável. Essa apreciável quantidade de papel pode ser reutilizada como matéria-prima de outros produtos, como ocorre em países como Estados Unidos e Japão. Ince e Mckeever (1995) descrevem que chapas isolantes, produtos moldados, chapas aglomeradas, chapas duras, material para assoalhos, coberturas e paredes de habitações são alguns exemplos do uso desses materiais reciclados nesses países.

Este trabalho de pesquisa teve como objetivo analisar a qualidade das chapas aglomeradas compostas por partículas de madeira de Pinus elliottii Engelm. associada à fragmentos de papel proveniente da coleta seletiva do lixo.

\section{REVISÃO DE LITERATURA}

Existe uma grande variedade de materiais que podem ser combinados para a produção de compósitos (combinação de dois ou mais materiais unidos por algum tipo de betume ou matriz). Dentre eles, podem-se citar fibras virgens ou recicladas (papel ou madeira), resíduos agrícolas (bagaço de cana, palha de arroz e de trigo etc.) e polímeros sintéticos como polipropileno, polistireno e polietileno.

Brito (1995), fazendo um estudo da viabilidade econômica do uso de resíduos de madeira para a produção de chapas aglomeradas, descreveu que a indústria nacional se tem utilizado quase que exclusivamente de madeira em toras de coníferas plantadas (pinus) para a fabricação desse tipo de chapa. Apesar do fato de que boa parte dos resíduos de madeira serem usados para a geração de energia e a disposição geográfica das serrarias e laminadoras brasileiras prejudicarem sua coleta e transporte, aqueles resíduos de madeira, que se encontram numa distância satisfatória das fábricas, devem ser vistos como uma fonte de matéria-prima complementar de baixo custo para a fabricação de aglomerados.

Akiyama et al. (1996) utilizaram fibras de papel recicláveis para confeccionar chapas que estão sendo utilizadas no Japão para a confecção de formas de concreto. Essas chapas de fibras demonstraram boa resistência à flexão, colagem interna e resistência à água. Foi provado também que as propriedades de resistência dessas chapas se mantêm constantes durante o curto período de tempo em que as formas de concreto são utilizadas.

Massijaya e Okuma (1996) fizeram um estudo objetivando a produção de painéis de alta qualidade a com base em papel jornal. Partículas de madeiras foram misturadas ao papel jornal em diferentes proporções e posições (ao acaso, nas faces ou no miolo). $\mathrm{O}$ aumento da percentagem de madeira no painel proporcionou melhora quanto à colagem interna e à estabilidade dimensional. Com relação ao módulo de ruptura (MOR) e ao módulo de elasticidade (MOE), os resultados demonstraram que a introdução do papel se mostrou benéfico.

Haselein et al. (2002) confeccionaram chapas aglomeradas utilizando gesso como material cimentante e papel reciclável dissociado (jornal e offset). Os autores observaram que a inclusão de fibras de papel causou aumento das propriedades mecânicas das chapas, tais como a resistência à flexão estática, dureza e arrancamento de parafusos, quando comparados ao tratamento testemunha (gesso puro). Já a inclusão de papel offset não causou diferença estatística significativa com relação à testemunha na maior parte das propriedades avaliadas. Concluíram ainda que foi possível a fabricação de chapas com papel reciclado com inchamento em espessura, após imersão em água por 24 horas, menor que $1 \%$.

Por outro lado, qualquer alteração na geometria ou granulometria das partículas impõem a necessidade de se averiguar a melhor proporção de adesivo a ser empregado, entre outras variáveis presentes no processo (Brito e Peixoto, 2000). Alterações do volume da manta de partículas são proporcionadas pelo uso de diferentes tipos e geometria de partículas. Quanto menor o volume do colchão, menor é a área de recebimento dos compostos em emulsão (adesivo, parafina e água) e, conseqüentemente, maiores são as 
perdas.

Dentre os adesivos utilizados para a confecção de painéis de madeira podemos citar os taninos, os quais são substâncias orgânicas de origem vegetal, mais precisamente fenóis e polifenóis. Portanto, trata-se de um adesivo proveniente de fontes naturais. Mori et al. (1999) descreveram que os taninos são produtos utilizados para curtir couro desde a antigüidade, sendo recente sua utilização para a fabricação de adesivo.

De acordo com Calegari et al. (2002), para a colagem de chapas aglomeradas com adesivo à base de tanino-formaldeído, altos teores de umidade do colchão são utilizados quando comparados às resinas fenólicas e uréicas. Por isso, explosões da chapa na abertura dos pratos da prensa podem ocorrer com maior frequiência, em consequiência da pressão de vapor d'água.

\section{MATERIAIS E MÉTODOS}

O presente trabalho foi realizado no Laboratório de Produtos Florestais, pertencente à Universidade Federal de Santa Maria (UFSM), Santa Maria, RS. Dois tipos de materiais lignocelulósicos foram utilizados para a confecção das chapas aglomeradas: partículas de madeira e papel reciclável dos tipos jornal e offset (ou sulfite).

A madeira para a produção das partículas foram obtidas de três exemplares de Pinus elliottii Engelm. de 15 anos de idade. De cada árvore, foram retiradas as duas primeiras toras de aproximadamente 2,5 $\mathrm{m}$ de comprimento, até a altura de $5 \mathrm{~m}$ da árvore. Discos foram retirados das mesmas posições para a determinação da densidade básica da madeira (peso seco/volume saturado).

Com uma serra-de-fita, as toras foram posteriormente transformadas em tábuas de espessura média de 19,1 mm, sendo, em seguida, armazenadas em um tanque com água. Saturadas, cada tábua foi secionada em seu sentido transversal em comprimento médio de $75 \mathrm{~mm}$ em serra circular. Ainda saturadas e a uma temperatura em torno de $50^{\circ} \mathrm{C}$, essas baguetas foram transformadas em flocos com espessura média de 0,75 $\mathrm{mm}$. Um tanque adaptado com aquecedor elétrico foi utilizado para o aquecimento das baguetas e um flaker de laboratório para a produção dos flocos. Desse modo, obteram-se flocos de comprimento, largura e espessura de aproximadamente $75 \times 19,1 \times 0,75 \mathrm{~mm}$. Após a secagem dos flocos, estes foram picados em moinho de martelo dotado com peneira com malhas de $8,0 \mathrm{~mm}$ de diâmetro. As partículas assim obtidas foram classificadas numa segunda peneira com malhas de $3 \times 3 \mathrm{~mm}$ para a retirada dos finos.

O papel jornal foi obtido pelo recolhimento de jornais circulantes no município de Santa Maria, RS. Já o papel offset utilizado foi obtido da Gráfica Universitária, UFSM. As aparas de ambos os tipos de papéis foram utilizadas tanto no formato de tiras quanto na forma de papel moído. Uma guilhotina manual para papéis foi utilizada para a confecção das tiras, as quais foram cortadas em dimensões nominais de $110 \mathrm{~mm}$ de comprimento e $20 \mathrm{~mm}$ de largura. O moinho de martelos com a mesma peneira que foi utilizada para a confecção das partículas de madeira também foi utilizada para a moagem dos papéis.

Aproximadamente 12 horas antes de sua utilização, o papel e as partículas de madeira foram colocados em estufa com circulação forçada de ar a $60^{\circ} \mathrm{C}$, a qual proporcionou um teor de umidade de aproximadamente $3 \%$ (base seca).

$\mathrm{Na}$ confecção das chapas, os dois tipos e formatos de papéis foram misturados às partículas de madeira em diferentes proporções, baseando-se no delineamento fatorial. Um tratamento testemunha também foi utilizado (Tabela 1).

As chapas aglomeradas foram produzidas aleatoriamente com três repetições por tratamento, com densidade nominal igual a $0,65 \mathrm{~g} / \mathrm{cm}^{3}$ (peso seco/volume ao teor de umidade de equilíbrio). O adesivo utilizado foi à base de tanino-formaldeído na proporção 100:10 respectivamente (parte por peso de sólidos nãovoláteis). Utilizou-se tanino em pó cedido pela empresa SETA S.A., localizada em Estância Velha, RS.

Sabendo-se que, em conseqüência da variação da geometria das aparas de papéis, ocorreria uma variação do teor de adesivo (teor de umidade) do colchão, o tanino em pó foi solubilizado em água na quantidade necessária para que essa mistura apresentasse um teor de sólido de 35\%. Estabeleceu-se essa percentagem de sólido uma vez que, segundo cálculos, esse valor proporcionaria à manta de partículas um teor da umidade de $16,8 \%$. Desse modo, não necessitaria a adição de água ao colchão o que dificultaria o controle do teor de adesivo baseado no teor de umidade. 
TABELA 1: Relação dos tratamentos utilizados na confecção das chapas.

TABLE 1: Treatments used for boards manufacturing.

\begin{tabular}{c|c|c|c}
\hline \multirow{2}{*}{ Tratamento } & Relação & \multicolumn{2}{|c}{ Papel } \\
\cline { 3 - 4 } papel:madeira & $0: 100$ & Tipo & Formato \\
\cline { 3 - 4 } 1-Testemunha & $20: 80$ & - & - \\
\hline 2 & $40: 60$ & jornal & moído \\
3 & $60: 40$ & jornal & moído \\
4 & $100: 0$ & jornal & moído \\
5 & $20: 80$ & jornal & moído \\
6 & $40: 60$ & jornal & tiras \\
7 & $60: 40$ & jornal & tiras \\
8 & $100: 0$ & jornal & tiras \\
9 & $20: 80$ & offset & moído \\
10 & $40: 60$ & offset & moído \\
11 & $60: 40$ & offset & moído \\
12 & $100: 0$ & offset & moído \\
13 & $20: 80$ & offset & tiras \\
14 & $40: 60$ & offset & tiras \\
15 & $60: 40$ & offset & tiras \\
16 & $100: 0$ & offset & tiras \\
\hline
\end{tabular}

O tanino solubilizado, com pH igual a 4,2, foi devidamente corrigido para 8,0 com o uso de hidróxido de sódio. A formalina, por sua vez, apresentava $40 \%$ de sólidos. Os teores de parafina (emulsão com $52 \%$ de sólidos) e adesivo foram mantidos constantes, utilizando-se 1 e $10 \%$ respectivamente, baseando-se no peso seco dos componentes formadores da chapa (partícula de madeira e papel reciclado e partículas sólidas de adesivo e parafina).

Os materiais em emulsão (adesivo e parafina) foram misturados às partículas de madeira e/ou papel com o auxílio de um tambor de laboratório dotado de uma pistola acionada por ar comprimido. A mistura foi espalhada manualmente em uma caixa de madeira de fundo falso e sem tampa, com dimensões 40 x 40 x $20 \mathrm{~cm}$, colocada sobre uma chapa de aço galvanizada com 1,0 mm de espessura e revestida com papel vegetal. Esse papel vegetal foi utilizado para dar um melhor acabamento às faces da chapa aglomerada e evitar sua aderência às chapas de aço. As partículas caíram por gravidade e foram orientadas ao acaso. Uma homogeneização manual foi realizada para melhorar a distribuição das partículas. Posteriormente sofreram uma pré-prensagem à temperatura ambiente durante um período aproximado de 2 minutos. Para cada colchão confeccionado, retiraram-se do tambor rotativo duas amostras da mistura para a determinação do seu teor de umidade.

O colchão de partículas foi então levado para uma prensa hidráulica, com pratos horizontais e aquecidos a $180^{\circ} \mathrm{C}$. Essa prensagem definitiva foi feita durante 10 minutos, a uma pressão manométrica de $180 \mathrm{kgf} / \mathrm{cm}^{2}$ (pressão específica aproximada de $45 \mathrm{kgf} / \mathrm{cm}^{2}$ ). A espessura final das chapas (estabelecida em $0,95 \mathrm{~cm}$ ) foi determinada com o auxílio de duas barras de aço colocadas em lados opostos ao colchão os quais limitavam o fechamento total dos pratos da prensa. Posteriormente, as chapas foram levadas à câmara climatizada onde foram mantidas a $20^{\circ} \mathrm{C}$ e $65 \%$ de umidade relativa até peso constante. Após ter tido suas bordas aparadas para melhorar seu manuseio, mediu-se a espessura média de cada chapa aglomerada.

Os corpos-de-prova (amostras) foram confeccionados após as chapas terem sido levemente lixadas. Utilizaram-se paquímetro digital e balança eletrônica para a determinação da densidade a qual foi determinada pela razão entre o peso seco ( $0 \%$ de umidade) e volume ao teor de umidade de equilíbrio.

Para a realização do teste de flexão estática, da resistência ao arrancamento de parafusos e da dureza Janka das chapas, utilizou-se uma máquina universal de ensaios, hidráulica, marca AMSLER. Utilizou-se o restante dos corpos-de-prova do teste de flexão para a realização dos dois últimos ensaios e para a 
determinação do teor de umidade das amostras utilizadas. A avaliação da absorção d'água e do inchamento em espessura foi realizada após 24 horas de imersão. Todos os testes foram realizados segundo a norma ASTM D 1037 (1995).

Os dados foram analisados por meio de análise de variância e regressão, relacionando as propriedades das chapas à percentagem, tipo e formato das aparas de papéis utilizadas, ao nível de 5\% de probabilidade.

\section{RESULTADOS E DISCUSSÕES}

A madeira utilizada apresentou densidade igual a $0,485 \mathrm{~g} / \mathrm{cm}^{3}$. As partículas oriundas desse material, assim como os papéis reciclados, que foram moídos, se apresentaram no formato aproximadamente retangular, com lados irregulares. As aparas, que foram secionadas em guilhotina, puderam ser confeccionadas no formato requerido. A Tabela 2 mostra as dimensões reais médias dos diferentes tipos de partículas utilizadas.

TABELA 2: Dimensões reais médias dos diferentes tipos de partículas utilizadas.

TABLE 2: Average size of the generated particles.

\begin{tabular}{c|c|c|c|c|c}
\hline \multirow{2}{*}{ Material } & \multirow{2}{*}{ Tipo } & \multirow{2}{*}{ Formato } & \multicolumn{3}{|c}{ Dimensões ${ }^{1}(\mathrm{~mm})$} \\
\cline { 4 - 6 } & & & Comprimento & Largura & Espessura \\
\hline \multirow{2}{*}{ madeira } & pinus & moído $^{2}$ & 10,8 & 2,8 & 0,72 \\
\multirow{2}{*}{ papel } & \multirow{2}{*}{ jornal } & tiras $^{3}$ & 107,8 & 20,6 & 0,08 \\
\cline { 3 - 6 } & \multirow{2}{*}{ offset } & moído $^{2}$ & 12,3 & 4,4 & 0,10 \\
\cline { 3 - 6 } & & tiras $^{3}$ & 109,6 & 20,0 & 0,10 \\
\cline { 3 - 6 } & & moído $^{2}$ & 11,2 & 5,4 & 0,12 \\
\hline
\end{tabular}

Em que: 1 = Média de aproximadamente cem partículas; 2 = Partículas de formato aproximadamente retangular com lados irregulares; 3 = Partículas de formato retangular, lados regulares.

Os painéis aglomerados produzidos com papéis no formato de tiras foram os mais problemáticos. Dois problemas relacionados especificamente pelo uso desse tipo de apara ocorreram: estouro das chapas durante a abertura dos pratos da prensa e depósito de partículas de madeira na face inferior do colchão.

$\mathrm{O}$ estouro foi mais pronunciado nas chapas produzidas pelo uso de tiras de papel offset. Esse problema não ocorreu em todas as repetições dos tratamentos que utilizavam esse tipo de partícula, sendo que algumas repetições puderam ser feitas sem que ocorresse esse problema. Em algumas chapas, as rachaduras internas podiam ser nitidamente observadas, uma vez que ocorria uma sobre elevação na face dos painéis, evidenciando que no seu interior existiam bolhas de ar. Em outras, no entanto, as rachaduras só puderam ser detectadas após a confecção dos corpos-de-prova. O segundo problema ocorreu durante a montagem da manta de partículas no molde de madeira. As partículas de madeira, por serem mais pesadas e pequenas quando comparadas com as tiras de papel, escorregavam entre os espaços vazios proporcionados pelas tiras e depositavam-se na face inferior do colchão. $\mathrm{O}$ agito usado para homogeneizar manualmente as partículas durante a fase de montagem da manta favoreceu o agravamento desse problema.

Em nenhum dos tratamentos baseados no uso de papel moído ocorreram os problemas de estouro e depósito de partículas de madeira em uma das faces. Entretanto, em todos os tratamentos, teve-se problema relativo ao teor de umidade do colchão. A combinação de diferentes tipos, formatos e mistura de materiais lignocelulósicos proporcionaram a formação de volumes diferenciados para cada tratamento estudado, ocasionando uma variação nas perdas dos componentes em emulsão no interior do tambor rotativo. A fim de evitar uma variação significativa na quantidade dos componentes em emulsão aplicados com relação a cada tratamento, procurou-se corrigir seus pesos com diferentes margens, por meio de tentativa, e baseando-se no teor de umidade do colchão determinado após a mistura. Em colchões de maiores volumes (tratamentos baseados no uso de papel jornal moído), menores margens foram necessárias quando comparados a colchões de menores volumes (tratamento testemunha). As chapas, que apresentaram umidade distinta da média requerida foram descartadas e confeccionadas novamente. Desse modo, conseguiu-se homogeneizar o teor de umidade dos colchões com relação aos tratamentos, como pode ser observado pela Tabela 3. 
TABELA 3: Valores médios reais das características das chapas com relação aos tratamentos.

TABLE 3: Average values of the board characteristics related to the treatments.

\begin{tabular}{ccc|c|c}
\hline Tratamento & Umidade do colchão ${ }^{2}(\%)$ & Umidade de equilíbrio $^{3}(\%)$ & Densidade $^{4}\left(\mathrm{~g} / \mathrm{cm}^{3}\right)$ \\
\hline 1 & $16,6 \mathrm{a}^{1}$ & $9,7 \mathrm{i}$ & $0,64 \mathrm{ab}$ \\
2 & $16,4 \mathrm{a}$ & $9,3 \mathrm{hi}$ & $0,65 \mathrm{abc}$ \\
3 & $17,2 \mathrm{a}$ & $9,2 \mathrm{gh}$ & $0,65 \mathrm{ab}$ \\
4 & $17,3 \mathrm{a}$ & $9,2 \mathrm{gh}$ & $0,63 \mathrm{ab}$ \\
5 & $16,4 \mathrm{a}$ & $8,8 \mathrm{ef}$ & $0,62 \mathrm{a}$ \\
6 & $16,1 \mathrm{a}$ & $9,6 \mathrm{i}$ & $0,65 \mathrm{bc}$ \\
7 & $17,2 \mathrm{a}$ & $9,2 \mathrm{~h}$ & $0,68 \mathrm{~cd}$ \\
8 & $17,4 \mathrm{a}$ & $9,1 \mathrm{fgh}$ & $0,72 \mathrm{e}$ \\
9 & $15,9 \mathrm{a}$ & $8,7 \mathrm{e}$ & $0,69 \mathrm{de}$ \\
10 & $16,4 \mathrm{a}$ & $9,3 \mathrm{~h}$ & $0,64 \mathrm{ab}$ \\
11 & $16,8 \mathrm{a}$ & $8,8 \mathrm{ef}$ & $0,64 \mathrm{ab}$ \\
12 & $16,6 \mathrm{a}$ & $8,3 \mathrm{~d}$ & $0,64 \mathrm{ab}$ \\
13 & $16,3 \mathrm{a}$ & $7,4 \mathrm{~b}$ & $0,65 \mathrm{ab}$ \\
14 & $17,2 \mathrm{a}$ & $8,9 \mathrm{efg}$ & $0,65 \mathrm{bc}$ \\
15 & $16,9 \mathrm{a}$ & $8,1 \mathrm{sd}$ & $0,69 \mathrm{de}$ \\
16 & $16,9 \mathrm{a}$ & $7,9 \mathrm{c}$ & $0,70 \mathrm{de}$ \\
17 & $16,4 \mathrm{a}$ & $6,1 \mathrm{a}$ & $0,75 \mathrm{f}$ \\
\hline
\end{tabular}

Em que: 1 = Valores seguidos pela mesma letra, na mesma coluna, não diferem estatisticamente, ao nível de $5 \%$ de probabilidade, pelo teste Least Significant Difference (LSD) de Fisher; $2=$ Base úmida; $3=$ Base úmida. T: $20^{\circ} \mathrm{C}$ e UR: $65 \%$; 4 = Razão entre peso seco e volume ao teor de umidade de equilíbrio.

Procurou-se manter constante a densidade das chapas durante a fabricação. No entanto, isso nem sempre foi possível, conforme indicado pela análise da variância, a qual demonstrou diferença estatística significativa entre os tratamentos (Tabela 3).

Já os valores médios das propriedades físicas e mecânicas das chapas, em função dos tratamentos, são apresentados na Tabela 4.

TABELA 4: Valores médios das propriedades físicas e mecânicas das chapas aglomeradas em função dos tratamentos.

TABLE 4: Average values of the physical and mechanical properties of the particleboards as a function of the treatments.

\begin{tabular}{c|c|c|c|c|c|c|c}
\hline \multirow{2}{*}{ Tratamento } & \multicolumn{2}{|c|}{ Propriedades Físicas } & \multicolumn{5}{c}{ Propriedades Mecânicas } \\
\cline { 2 - 8 } & $\begin{array}{c}\text { Abs.24H } \\
(\%)\end{array}$ & $\begin{array}{c}\text { Inch.24H } \\
(\%)\end{array}$ & $\begin{array}{c}\mathrm{MOE}^{3} \\
\left(\mathrm{kgf} / \mathrm{cm}^{2}\right)\end{array}$ & $\begin{array}{c}\mathrm{MOR}^{4} \\
\left(\mathrm{kgf} / \mathrm{cm}^{2}\right)\end{array}$ & $\begin{array}{c}\mathrm{LI}^{5} \\
\left(\mathrm{kgf} / \mathrm{cm}^{2}\right)\end{array}$ & $\begin{array}{c}\text { R.A.P } \\
(\mathrm{kgf})\end{array}$ & $\begin{array}{c}\text { Dureza } \\
(\mathrm{kgf})\end{array}$ \\
\hline 1 & 55,0 & 15,6 & 16056 & 142,8 & 5,72 & 105,0 & 348,4 \\
2 & 80,3 & 18,0 & 13019 & 145,2 & 3,02 & 88,5 & 375,0 \\
3 & 92,9 & 20,4 & 12642 & 154,1 & 1,88 & 77,2 & 301,5 \\
4 & 99,9 & 22,7 & 7706 & 138,1 & 1,71 & 65,4 & 301,5 \\
5 & 125,0 & 38,3 & 5540 & 131,6 & 1,21 & 50,7 & 189,7 \\
6 & 71,2 & 22,2 & 10525 & 125,4 & 1,88 & 85,5 & 329,8 \\
7 & 71,2 & 23,1 & 14006 & 128,0 & 1,51 & 96,8 & 401,1 \\
8 & 76,6 & 28,7 & 17489 & 168,3 & 1,43 & 108,3 & 452,7 \\
\hline 9 & 107,2 & 38,3 & 17069 & 168,3 & 0,69 & 78,5 & 371,0 \\
10 & 90,5 & 18,2 & 9573 & 111,3 & 2,68 & 91,8 & 289,0 \\
11 & 96,3 & 20,6 & 6378 & 112,9 & 2,25 & 69,7 & 216,7 \\
12 & 106,5 & 22,7 & 3232 & 130,0 & 1,57 & 53,8 & 186,7 \\
13 & 115,6 & 19,2 & 2461 & 149,2 & 1,24 & 33,7 & 93,8 \\
\hline
\end{tabular}


TABELA 4: Continuação ...

TABLE 4: Continued ...

\begin{tabular}{|c|c|c|c|c|c|c|c|}
\hline \multirow[b]{2}{*}{ Tratamento } & \multicolumn{2}{|c|}{ Propriedades Físicas } & \multicolumn{5}{|c|}{ Propriedades Mecânicas } \\
\hline & $\begin{array}{c}\text { Abs.24H }{ }^{1} \\
(\%)\end{array}$ & $\begin{array}{c}\text { Inch.24H }{ }^{2} \\
(\%)\end{array}$ & $\begin{array}{c}\mathrm{MOE}^{3} \\
\left(\mathrm{kgf} / \mathrm{cm}^{2}\right)\end{array}$ & $\begin{array}{c}\begin{array}{c}\mathrm{MOR}^{4} \\
\left(\mathrm{kgf} / \mathrm{cm}^{2}\right)\end{array} \\
\end{array}$ & $\begin{array}{c}\mathrm{LI}^{5} \\
\left(\mathrm{kgf} / \mathrm{cm}^{2}\right)\end{array}$ & $\begin{array}{r}\text { R.A.P } \\
\text { (kgf) }\end{array}$ & $\begin{array}{c}\text { Dureza }^{7} \\
(\mathrm{kgf})\end{array}$ \\
\hline 14 & 81,3 & 28,4 & 11334 & 118,5 & 0,97 & 85,5 & 309,5 \\
\hline 15 & 83,7 & 31,9 & 8456 & 123,1 & 0,81 & 88,2 & 229,4 \\
\hline 16 & 90,8 & 28,1 & 9414 & 124,1 & 0,98 & 88,2 & 288,3 \\
\hline 17 & 82,5 & 27,7 & 2897 & 183,6 & 0,54 & 50,8 & 97,8 \\
\hline
\end{tabular}

Em que: 1 = Absorção d'água após 24 horas de imersão; 2 = Inchamento em espessura após 24 horas de imersão em água; 3 = Módulo de elasticidade; 4 = Módulo de ruptura; 5 = Ligação interna; $6=$ Resistência ao arrancamento de parafusos; 7 = Dureza Janka.

Relacionando os valores do inchamento em espessura com a norma DIN 68761 (1)-1961, (3)-1971, observou-se que somente o tratamento 1 (testemunha) satisfez essa norma. Todas as inclusões de aparas provocaram aumento em inchamento inaceitável. Isso também ocorreu com relação à ligação interna. Baseando-se na norma americana ANSI A 208.1 (1987), todos os valores de MOR encontrados foram aceitáveis, enquanto que para o MOE, somente os tratamentos 8 e 9 atenderam à exigência. Para o teste de dureza, somente alguns tratamentos (tratamentos 5, 11, 12, 13 e 17) não atingiram o valor mínimo exigido. Pela mesma norma, somente os tratamentos 1 e 8 se mostraram aceitáveis com relação à resistência ao arrancamento de parafusos.

Modelos significativos foram encontrados para todas as propriedades analisadas, conforme mostra a Tabela 5 e Figuras 1 a 7.

TABELA 5: Equações ajustadas para as propriedades das chapas.

TABLE 5: Adjusted equations for the board properties.

\begin{tabular}{|c|c|c|c|c|c|}
\hline Propriedade & $\begin{array}{c}\text { Tipo e Formato } \\
\text { do Papel }\end{array}$ & Equação Ajustada ${ }^{1}$ & $\mathrm{~F}$ & Syx \% & $\begin{array}{c}\mathrm{R}^{2} \\
\text { Ajustado } \\
(\%)\end{array}$ \\
\hline \multirow{4}{*}{$\begin{array}{c}\text { Absorção } \\
\text { d'água } \\
24 \mathrm{H}\end{array}$} & pap. $1^{2}$ e par. $1^{4}$ & $198,247+1,0253 \mathrm{P}-0,003931 \mathrm{P}^{2}-228,566 \mathrm{~d}$ & 340,3 & 3,9 & 91,1 \\
\hline & pap. 1 e par. 2 & $165,705+0,7139 P-0,001098 P^{2}-173,155 d$ & 72,0 & 6,1 & 68,1 \\
\hline & pap. $2^{3}$ e par. 1 & $206,038+1,3406 \mathrm{P}-0,007355 \mathrm{P}^{2}-238,488 \mathrm{~d}$ & 403,7 & 3,4 & 92,5 \\
\hline & pap. 2 e par. $2^{5}$ & $135,004+1,18658 \mathrm{P}-0,007697 \mathrm{P}^{2}-122,97 \mathrm{~d}$ & 77,6 & 4,4 & 69,5 \\
\hline \multirow{4}{*}{$\begin{array}{c}\text { Inchamento } \\
\text { em } \\
\text { espessura } \\
24 \mathrm{H}\end{array}$} & pap. 1 e par. 1 & $5,4301+0,10515 \mathrm{P}+0,000204 \mathrm{P}^{2}+16,691 \mathrm{~d}$ & 145,2 & 1,1 & 81,2 \\
\hline & pap. 1 e par. 2 & $1,4863+0,1263 \mathrm{P}+0,00075 \mathrm{P}^{2}+23,847 \mathrm{~d}$ & 84,6 & 2,3 & 71,5 \\
\hline & pap. 2 e par. 1 & $-3,638+0,2080 P-0,001731 P^{2}+30,5875 d$ & 49,9 & 1,1 & 59,2 \\
\hline & pap. 2 e par. 2 & $-18,963+0,4023 \mathrm{P}-0,004035 \mathrm{P}^{2}+58,537 \mathrm{~d}$ & 69,1 & 1,9 & 67,2 \\
\hline \multirow{4}{*}{ MOE } & pap. 1 e par. 1 & $-19537-158,883 \mathrm{P}+0,6732 \mathrm{P}^{2}+55746,4 \mathrm{~d}$ & 167,8 & 1035,2 & 83,4 \\
\hline & pap. 1 e par. 2 & $-2552,84-70,626 P+0,8933 P^{2}+26933,6 d$ & 8,2 & 2703,7 & 17,6 \\
\hline & pap. 2 e par. 1 & $-15798,8-326,90 P+1,9796 P^{2}+49813,3 d$ & 258,4 & 1022,1 & 88,4 \\
\hline & pap. 2 e par. 2 & $-10595,5-201,085 \mathrm{P}+0,4530 \mathrm{P}^{2}+40476,6 \mathrm{~d}$ & 89,6 & 1397,4 & 72,9 \\
\hline \multirow{4}{*}{ MOR } & pap. 1 e par. 1 & $-179,36-0,0732 \mathrm{P}+0,001 \mathrm{P}^{2}+503,678 \mathrm{~d}$ & 56,7 & 8,0 & 62,8 \\
\hline & pap. 1 e par. 2 & $-41,4676+0,0299 P+0,0027 P^{2}+275,14 d$ & 14,4 & 22,1 & 28,5 \\
\hline & pap. 2 e par. 1 & $-220,557-0,912 P+0,0113 P^{2}+561,017 d$ & 15,3 & 18,0 & 60,5 \\
\hline & pap. 2 e par. 2 & $10,0659-1,48083 \mathrm{P}+0,0176 \mathrm{P}^{2}+200,24 \mathrm{~d}$ & 37,1 & 12,1 & 52,3 \\
\hline \multirow{4}{*}{$\begin{array}{l}\text { Ligação } \\
\text { Interna }\end{array}$} & pap. 1 e par. 1 & $1,4499-0,1051 \mathrm{P}+0,000671 \mathrm{P}^{2}+5,8114 \mathrm{~d}$ & 152,5 & 0,6 & 88,5 \\
\hline & pap. 1 e par. 2 & $3,4517-0,1197 \mathrm{P}+0,0007995 \mathrm{P}^{2}+2,3961 \mathrm{~d}$ & 245,3 & 0,4 & 78,2 \\
\hline & pap. 2 e par. 1 & $0,85901-0,1035 P+0,00063 P^{2}+6,7801 d$ & 571,2 & 0,3 & 89,3 \\
\hline & pap. 2 e par. 2 & $4,4476+0,081947 \mathrm{P}-1,31158 \sqrt{ }(\mathrm{P})+1,737 \mathrm{~d}$ & 1116,2 & 0,3 & 94,3 \\
\hline
\end{tabular}


TABELA 5: Continuação ...

TABLE 5: Continued ...

\begin{tabular}{c|c|c|r|r|c}
\hline Propriedade & $\begin{array}{c}\text { Tipo e Formato } \\
\text { do Papel }\end{array}$ & Equação Ajustada ${ }^{1}$ & $\mathrm{~F}$ & Syx \% & $\begin{array}{c}\mathrm{R}^{2} \\
\text { Ajustado } \\
(\%)\end{array}$ \\
\hline Arranca- & pap. 1 e par. 1 & $-71,0727-0,9791 \mathrm{P}+0,00548 \mathrm{P}^{2}+274,30 \mathrm{~d}$ & 11,4 & 18,8 & 53,7 \\
mento & pap. 1 e par. 2 & $-68,8159-0,3047 \mathrm{P}-0,00046 \mathrm{P}^{2}+267,27 \mathrm{~d}$ & 8,4 & 22,7 & 42,5 \\
de & pap. 2 e par. 1 & $-68,6433-1,04758 \mathrm{P}+0,003329 \mathrm{P}^{2}+276,556$ & 1116,2 & 14,7 & 94,3 \\
parafusos & d & & & & \\
\hline & pap. 2 e par. 2 & $-90,826-0,4962 \mathrm{P}-0,00189 \mathrm{P}^{2}+292,328 \mathrm{~d}$ & 7,0 & 21,8 & 38,5 \\
\hline Dureza & pap. 1 e par. 1 & $-93,8049-1,173 \mathrm{P}-0,00272 \mathrm{P}^{2}+692,316 \mathrm{~d}$ & 118,5 & 18,6 & 78,3 \\
Janka & pap. 1 e par. 2 & $-436,45+1,6805 \mathrm{P}-0,01816 \mathrm{P}^{2}+1187,99 \mathrm{~d}$ & 8,1 & 103,9 & 42,2 \\
& pap. 2 e par. 1 & $-254,334-2,7430 \mathrm{P}+0,00462 \mathrm{P}^{2}+921,18 \mathrm{~d}$ & 28,5 & 74,7 & 74,7 \\
& pap. 2 e par. 2 & $-460,782-3,01113 \mathrm{P}+1247,86 \mathrm{~d}$ & 10,2 & 96,7 & 38,8 \\
\hline
\end{tabular}

Em que: $1=\mathrm{P}=$ percentagem de papel e $\mathrm{d}=$ densidade da chapa aglomerada; $2=$ Papel tipo 1 (jornal); $3=$ Papel tipo 2 (offset); 4 = Partícula formato 1 (moído); 5 = Partícula formato 2 (tiras).

Analisando-se as curvas ajustadas para as propriedades físicas (Figuras 1 e 2), observa-se que ocorre um gradual aumento da absorção d'água após 24 horas de imersão pela introdução de aparas de papel à chapa aglomerada. Esse aumento ocorre de maneira aproximadamente uniforme, independente do tipo e do formato do papel introduzido. Esse resultado coincide com Haselein et al. (2002), os quais observaram que não ocorre diferença estatística significativa na absorção d'água após 24 horas, quando diferentes tipos de papéis (jornal e offset) são introduzidos em chapas de partícula liga de gesso. O mesmo comportamento não ocorre com relação ao inchamento em espessura. Observa-se que o aumento da percentagem de papel offset introduzido causa um aumento gradual no inchamento até determinado limite (aproximadamente 60\%), a partindo do qual ocorre um decréscimo. Quando o papel jornal é utilizado, o aumento da sua percentagem na chapa ocasiona um aumento proporcional no inchamento, notadamente quando este se apresenta no formato de tiras. Haselein et al. (2002), no entanto, analisando apenas a mistura na razão madeira: gesso $=0,25$, não encontraram diferença estatística significativa dessa propriedade por causa do uso de diferentes tipos de papéis. Melhoria na estabilidade dimensional relacionada à maior percentagem de partícula de madeira, quando combinada com o papel jornal, já havia sido observada por Massijaya e Okuma (1996).

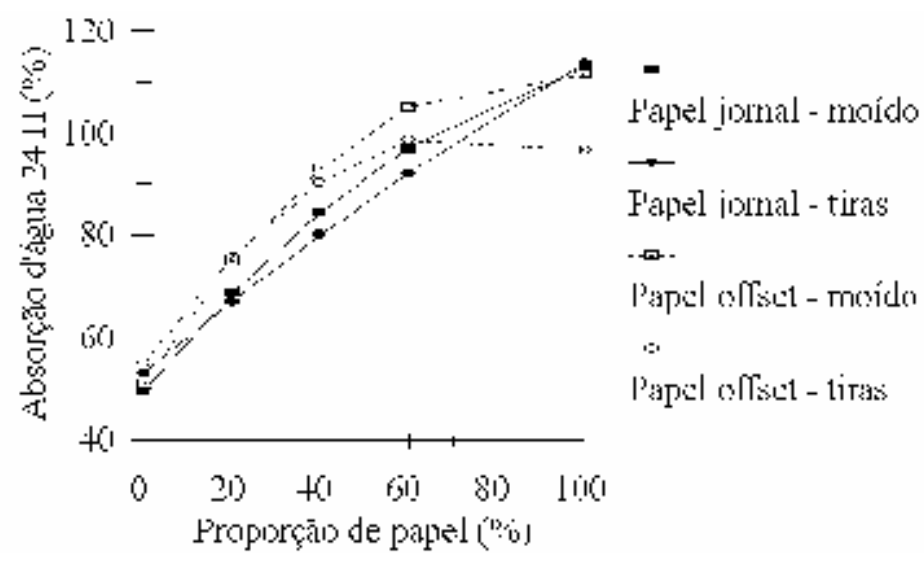

FIGURA 1: Curvas ajustadas para absorção d'água após 24 horas em função do tipo, formato e percentagem de papel analisado.

FIGURE 1: Fitted curve for water absorption after 24 hours as a function of type, shape and proportion of paper. 


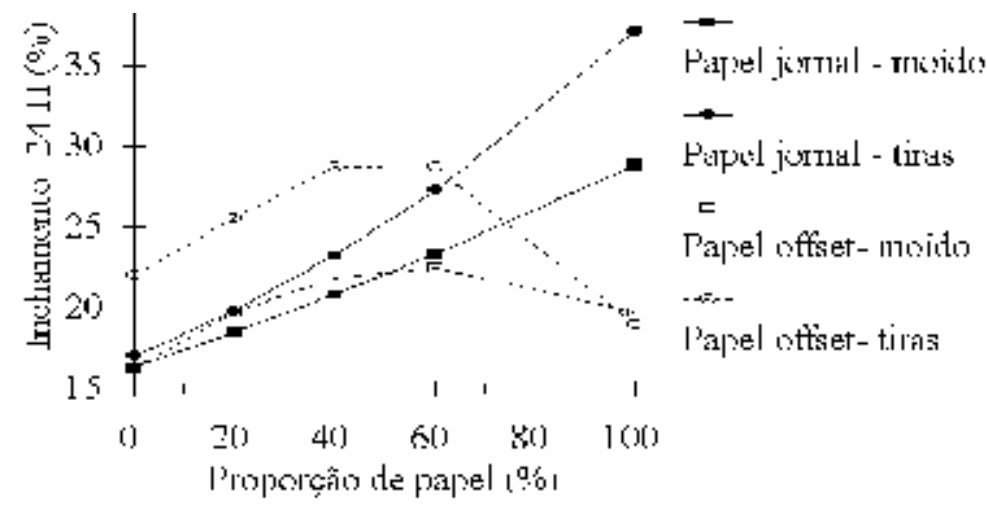

FIGURA 2: Curvas ajustadas para o inchamento em espessura após 24 horas em função do tipo, formato e percentagem de papel analisado.

FIGURE 2: Fitted curve for thickness swelling after 24 hours as a function of type, shape and proportion of paper.

As curvas ajustadas das propriedades relacionadas à flexão estática são mostradas na Figuras 3 e 4.

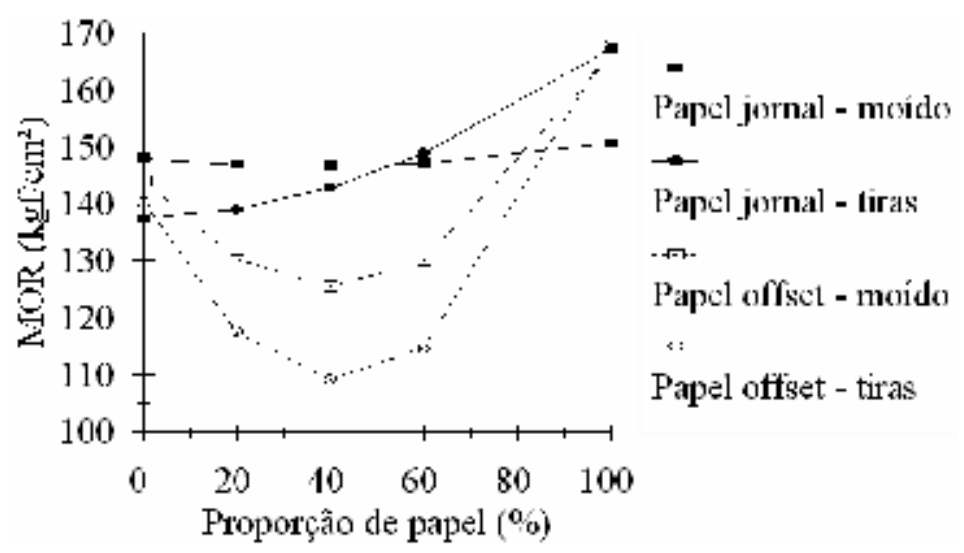

FIGURA 3: Curvas ajustadas para o MOR em função do tipo, formato e percentagem de papel analisado. FIGURE 3: Fitted curve for MOR as a function of type, shape and proportion of paper.

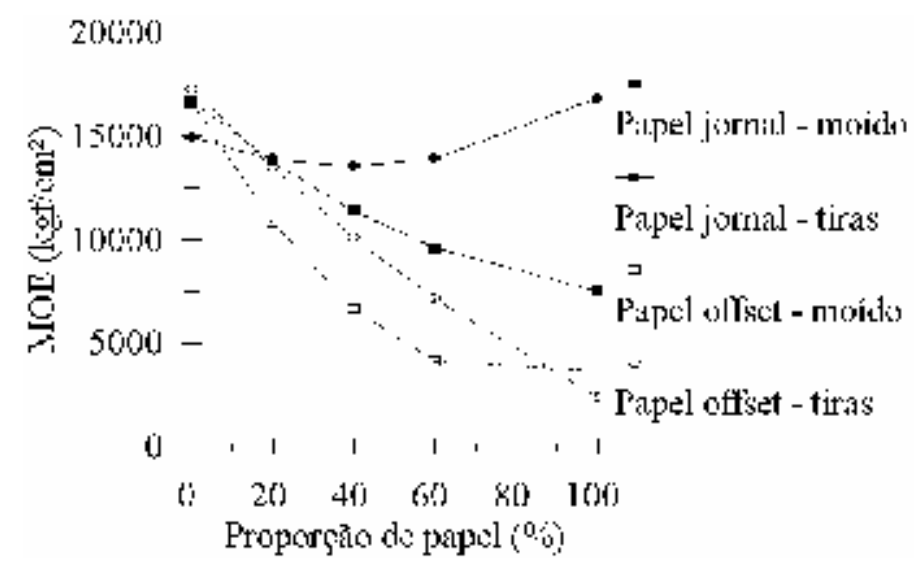

FIGURA 4: Curvas ajustadas para o MOE em função do tipo, formato e percentagem de papel analisado. FIGURE 4: Fitted curve for MOE as a function of type, shape and proportion of paper. 
Para o MOR, o aumento da percentagem de papel jornal ao painel aglomerado proporcionou melhoria dessa propriedade quando utilizado no formato de tiras. A inclusão de papel offset até $40 \%$ do peso do painel causou um decréscimo do MOR. Partindo desse limite, essa propriedade melhorou proporcionalmente ao aumento da percentagem de papel.

$\mathrm{O}$ aumento da percentagem de papel tipo jornal e na forma de tiras foi o único tratamento que proporcionou pequeno aumento do MOE (Figura 4). O aumento da percentagem dos demais tipos e formatos de papéis causou decréscimo dessa propriedade. De modo geral, observa-se que o aumento na inclusão de papel offset foi o que proporcionou maiores decréscimos dos valores do MOE.

Comparando essas propriedade relacionadas à flexão estática com os dados obtidos por Massijaya e Okuma (1996), verifica-se que o MOR se comporta de modo semelhante ao citado por esses autores. Entretanto, o MOE se comportou de maneira bastante diferenciada. Haselein et al. (2002) também verificaram melhor desempenho do MOR pelo uso de papel jornal quando comparado ao papel offset.

$\mathrm{O}$ aumento da percentagem de qualquer dos tipos e formato de papel causou redução dos valores de ligação interna (Figura 5). Reduções mais pronunciadas são verificadas pelo uso dos papéis no formato de tiras. O comportamento da curva pelo uso de papel moído, tanto jornal quanto offset, se mostrou semelhante, correspondendo a reduções menos drásticas em função do aumento da percentagem de papel. Massijaya e Okuma (1996), em seu trabalho, também verificaram que o aumento da percentagem de madeira proporciona melhoria da ligação interna.

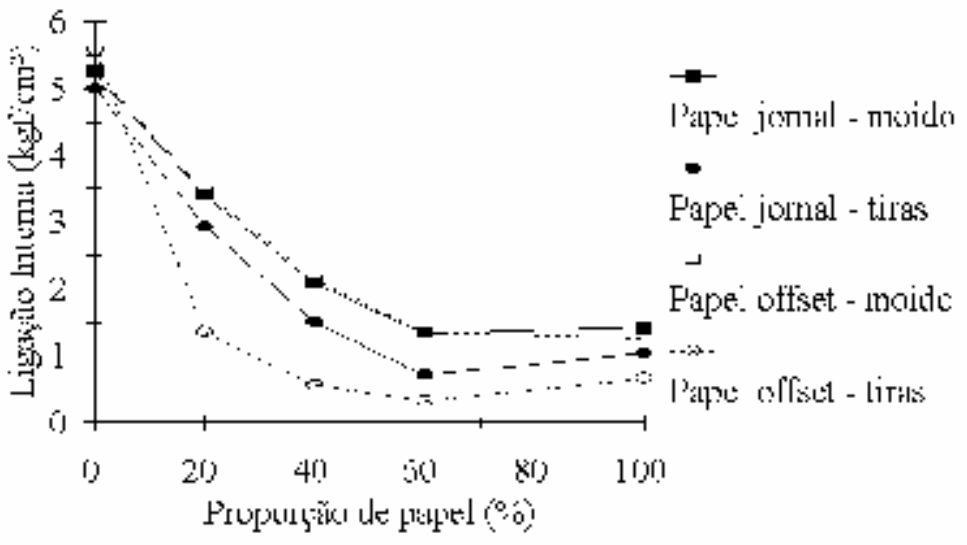

FIGURA 5: Curvas ajustadas para ligação interna em função do tipo, formato e percentagem de papel analisado.

FIGURE 5: Fitted curve for internal bond as a function of type, shape and proportion of paper.

A resistência ao arrancamento de parafusos também se mostrou prejudicada pela inclusão de papel (Figura 6). O melhor resultado foi proporcionado pelo uso de papel jornal quando utilizado no formato de tiras. Esse tratamento também demonstrou melhor resultado com relação ao teste de dureza (Figura 7). A inclusão de papel offset, em ambos os formatos, demonstrou os piores desempenhos. Da mesma forma, Haselein et al. (2002) observaram uma maior dureza proporcionada pelo uso de papel jornal quando comparado ao papel offset.

Os diferentes comportamentos nas propriedades físico-mecânicas observados nos painéis possivelmente estão relacionados aos diferentes processos de produção dos papéis. O processo mecânico de desfibramento geralmente utilizado para a produção do papel jornal provoca a ruptura da parede celular, expondo as microfibrilas e, conseqüentemente, aumentando a absorção d'água. Já o processo químico utilizado para a produção do papel offset decompõem parte da hemicelulose e, sobretudo a lignina, reduzindo a absorção d'água pelas microfibrilas quando comparado ao papel jornal. Adicionalmente, durante o processo 
de produção de papel offset, maior quantidade de cola é aplicada, o que reduz ainda mais sua higroscopicidade.

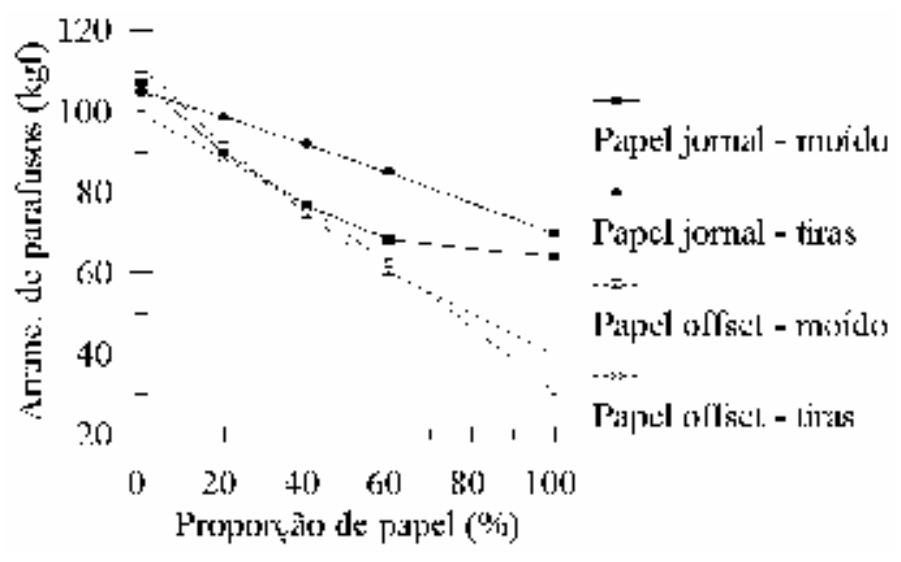

FIGURA 6: Curvas ajustadas para resistência ao arrancamento de parafusos em função do tipo, formato e percentagem de papel analisado.

FIGURE 6: Fitted curve for screw withdrawal as a function of type, shape and proportion of paper.

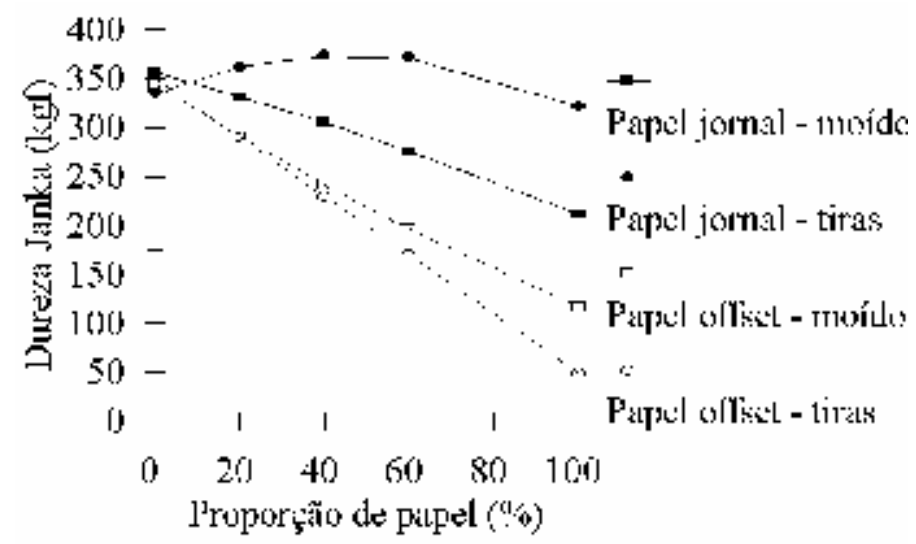

FIGURA 7: Curvas ajustadas para a dureza Janka em função do tipo, formato e percentagem de papel analisado.

FIGURE 7: Fitted curve for hardness as a function of type, shape and proportion of paper.

As diferentes gramaturas apresentadas pelos diferentes tipos de papéis também pode ter influenciado nos resultados. Para o papel jornal, que apresenta menor gramatura, foi necessário um maior volume do que o papel offset para satisfazer o peso necessário. Desse modo, os espaços vazios existentes entre as partículas de madeira foram melhor preenchidas quando comparadas ao papel offset.

\section{CONCLUSÕES}

Os resultados obtidos neste estudo permitem as seguintes conclusões:

Painéis de partículas aglomeradas produzidas tanto por aparas de papel jornal quanto offset no formato de tiras apresentaram problemas de estouro durante a abertura dos pratos da prensa e depósito de partículas de madeira na face inferior do colchão.

As inclusões de aparas de papel prejudicaram a maioria das propriedades analisadas, fazendo com que não atendessem às normas DIN 68761 (1)-1961, (3)-1971 e ANSI A 208.1 (1987). 
$\mathrm{O}$ aumento da percentagem de qualquer dos tipos e formato de aparas de papel causou maior prejuízo às propriedades de absorção e inchamento em espessura após 24 horas de imersão em água, ligação interna e resistência ao arrancamento de parafusos.

Pesquisas adicionais devem ser conduzidas utilizando pequenas quantidades de papéis recicláveis, assim como outros parâmetros de produção, visando a estabelecer a proporção de papel que poderia ser usada sem causar prejuízo para as propriedades das chapas.

\section{REFERÊNCIAS BIBLIOGRÁFICAS}

AKIYIMA,H.; KURIHAMA,K.; HOSOYA,S.; TOMIMURA,Y. Ecology boards for concrete forms using recycled paper resources. The use of recycled wood and paper in building applications. Madison: Forest Products Society, 1996. p.266.

AMERICAN NATIONAL STANDARDS INSTITUTE. Particleboard: ANSI A208.1. New York, 1987.

AMERICAN SOCIETY FOR TESTING AND MATERIALS. Standard test methods for avaluating properties of wood-base fiber and particle panel materials: D 1037-93. Philadelphia, PA, 1995.

BRITO,E.O. Estimativa da produção de resíduos na indústria brasileira de serraria e laminação de madeira. Revista da Madeira, Curitiba, v.9, n. 26, p.34-39, 1995.

BRITO,E.O.; PEIXOTO G.L. Avaliação da granulometria de partículas de Pinus taeda combinadas com adesivos comerciais para a fabricação de aglomerados. Revista Floresta e Ambiente, Rio de Janeiro, v.7, n.1, p. 60-67, 2000.

CALDERONI,S. O lixo que vira ouro. Revista Globo Ciência. São Paulo, n.85, p.26-31, 1998.

CALEGARI,L.; HASELEIN, C.R.; HILLIG, E.; SANTINI, E.J. Elevação da temperatura durante a prensagem de painéis aglomerados colados com tanino-fenol-formaldeído. In: CONGRESSO FLORESTAL ESTADUAL DO RIO GRANDE DO SUL, 8., 2000, Nova Prata-RS. Anais... Santa Maria: UFSM, 2002. p. 646-650.

CENCIG,E.G.S. A arte de reciclar. Disponível em: <http://www.geocities.com/RainForest/ Vines/4715/reciclagem.htm > . Acesso em: out. 2001.

GERMAN STANDARDS CIMMITTEE: Deutschen Normanausschuss. Specifications for particleboards. Holtz, 1971. (DIN 68761 (1)-1961 (3)).

HASELEIN,C.R.; CALEGARI, L.; ALBERTI, L.F.; MINELLO,A.L.; SILVA,P.A.; PINTOS, R.G.S. Fabricação de chapas de partículas aglomeradas usando gesso como material cimentante. Ciência Florestal, Santa Maria, v.12, n.1, p.81-88, 2002.

INCE,P.J.; MCKEEVER,D.B. Estimates of paper and wood recovery for recycling and potential for additional recovery in the United States. In: WOODFIBER plastic composites: virgin and recycled wood fiber and polymers for composites . Madison: Forest Products Society, 1995. p.144-154.

MASSIJAYA,M.Y; OKUMA,M.. Effect of wood particle mixing levels and board constructions on the properties of waste newspaper particleboard. In: THE USE of recycled wood and paper in building applications. Madison: Forest Products Society, 1996. p.211.

MORI,F.A.; VITAL,B.R.; DELLA LUCIA, R.M.; VALENTE,O.F.; PIMENTA, A.S. Utilização de resina à base de tanino da casca de Eucalyptus grandis W. Will ex Maiden na produção da painéis compensados. Revista Árvore, Viçosa, MG, v. 23, n.4, p.455-461, 1999.

SILVA,R.P.; OLIVEIRA,R.C. A reciclagem do papel: uma abordagem técnica. Folha Florestal,. Viçosa, MG, n. 93, p. 09-11, 1999. 